\title{
ACTIVATION ENERGIES OF THERMAL ANNEALING OF RADIATION-INDUCED DAMAGE IN n- AND p-CHANNELS OF CMOS INTEGRATED CIRCUITS
}

\author{
Vitaly Danchenko and E. G. Stassinopoulos \\ NASA, Goddard Space Flight Center \\ Greenbelt, Maryland 20771 \\ P. H. Fang \\ Department of Physics \\ Boston College \\ Chestnut Hill, Massachusetts 02167
}

\author{
Sidney S. Brashears \\ Space Science Services \\ Laurel, Maryland 20810
}

\begin{abstract}
$\underline{\text { Abstract }}$
Tempering and isothermal curves of annealing of radiation damage in p- and n-channels of both commercial, or "soft," and radiationhardened, or J-process, samples of RCA CD4007A CMOS integrated circuits, irradiated with both Co-60 gamma-rays and $1 \mathrm{MeV}$ electrons, have been determined. These experimental data were analyzed for activation energies of thermal annealing using two theoretical treatments, one of which is a new approach proposed here. The resulting activation energy distribution of p-channels of both the commercial and $\mathrm{J}$-process exhibit a single peak centered at about $1 \mathrm{eV}$, whereas the distributions of n-channels of the commercial process exhibit two distinct peaks centered at about 0.9 and $1.2 \mathrm{eV}$. The activation energy distributions of $n$-channels of the J-process show three peaks centered at about $0.7,1.0$ and $1.3 \mathrm{eV}$. The two peaks in the n-channels of the commercial devices are attributed to the double diffusion of phosphorus and boron in the formation of the p-well and the subsequent growth of the gate oxide using this silicon surface. If this reasoning is correct, then it follows that the radiation-induced charge trapping in the gate oxide occurs mainly around the impurity centers. The n-channels of the J-process exhibit considerable reverse annealing under elevated temperatures and large long-term room-temperature annealing as compared to p-channels. No differences in the annealing modes between devices irradiated with $\mathrm{Co}-60$ gamma rays and $1 \mathrm{MeV}$ electrons were observed.
\end{abstract}

\section{Introduction}

Thermal annealing of radiation-induced damage in semiconductor devices has become of increasing interest for space applications as projected mission lifetimes have increased and relatively permanent orbiting structures are being planned. Also, measurements and analyses of thermal annealing processes are useful tools for shedding light on the nature of charge carrier traps in semiconductors. In MOS structures, for example, thermal annealing can be used to reveal the nature of charge trapping in the gate insulators and insulator-semiconductor interface and to measure activation energies necessary to overcome potential barriers for electron-hole recombination.

Annealing of radiation damage in CMOS and other semiconductor devices may be roughly, but conveniently divided into three categories:

(1) Short-Term Annealing (room temperature and below) ${ }^{1-5}$

(2) Long-Term Annealing (room temperature) ${ }^{3}$ and

(3) Thermal Annealing (elevated temperatures). ${ }^{6-9}$

The first category usually refers to all the annealing processes that occur within a few minutes after irradiation. The study of these processes is important in the investigation of radiation damage, but is of very little benefit to space flight applications where the radiation rates are relatively low. The second category is of considerable practical importance for space flight, since the temperature around the spacecraft's hardware is usually controlled close to room temperature throughout the mission lifetime, which may be several years. This type of annealing of radiation damage often acts as a balancing agent in device radiation effects, bringing a device to a steady-state condition where radiation damage rates become equal to annealing rates. In some types of radiation-hard manufacturing processes the rate of annealing of radiation damage occurs even faster than the defect introduction rate. (This type of annealing, however, should not be confused with radiation-rate-dependent effects which are altogether different processes.) In some applications, where the amount of shielding allowed aboard the spacecraft approaches the borderline case, it is extremely important to be able to predict the amount of annealing that an engineer can count on in designing spacecraft electronics. The simplest way, of course, would be to measure the extent of annealing for the lifetime of an entire mission, but this is entirely impractical. It is, therefore, the approach of this program to use isothermal and tempering curves and the analysis of their accelerated annealing at elevated temperatures to derive the activation energy spectrum. Since the activation energy spectrum contains a relation between the time and the temperature dependence of the instability of the MOS devices, one should be able to evaluate and predict the behavior of these devices under a continuous variation of radiation and temperature, such as that which one encounters in the space radiation environment.

In earlier work, ${ }^{6,8}$ an analysis of isochronal and isothermal annealing curves for $\mathrm{p}$-channel MOS devices has revealed a distribution in activation energies, rather than a single activation energy. In this work, we have extended the analysis to include both p- and n-channels in a CMOS structure. In addition, we have introduced tempering, rather than isochronal curves for activation energy analysis, since tempering curves are more amenable to this kind of analysis. (In tempering, temperature is increased linearly as a function of time.) Also, a new approach to the theoretical analysis for activation energies is introduced.

\section{Experimental}

\section{CMOS Integrated Circuits}

The irradiation and annealing described in this work were performed on RCA CD4007A CMOS integrated circuits of two technologies: (1) a rad-soft, commercial process and (2) a rad-hard process, ${ }^{10,11}$ designated J-process. According to the manufacturer, the only difference between the two processes in the gate oxide is the nitrogen anneal temperature: the nitrogen anneal of the commercial process took place at $1100^{\circ} \mathrm{C}$, whereas that of the J-process took place at $850^{\circ} \mathrm{C}$. These devices consist of three n-channel and three p-channel enhancementmode MOS transistors. The oxide thickness is approximately $90 \mathrm{~nm}$ and is fabricated using the wet-oxide process. Typical gate threshold voltages are 1.8 to $2.0 \mathrm{~V}$ (n-channels) and -1.6 to $-1.8 \mathrm{~V}$ (p-channels). Gate threshold voltage in this work is defined as that voltage necessary to produce a drain current of $10 \mu \mathrm{A}$ with $10 \mathrm{~V}$ drain-to-substrate voltage. All ICs of each type came from the same wafer, made in this way 
by RCA specifically for the CREM* program, and are, therefore, spaceflight qualified.

$\underline{\text { Irradiation }}$

Irradiation was carried out in the Radiation Facility of Goddard Space Flight Center using both the $2 \mathrm{MeV}$ Van de Graaff accelerator and the Co-60 gamma-ray source. Since the commercial devices are much softer than the J-process devices, all the commercial ICs were irradiated to an average total fluence of $2.8 \times 10^{11} \mathrm{particles} / \mathrm{cm}^{2}$ in the case of electrons, or $10^{4} \mathrm{rad}-\mathrm{Si}$ in the case of gamma rays. J-process devices were irradiated with electrons to an average total fluence of $2.8 \times 10^{12}$ particles $/ \mathrm{cm}^{2}$, or $10^{5} \mathrm{rad}-\mathrm{Si}$ with gamma rays. The electron energy was $1 \mathrm{MeV}$, and the incident electron flux was measured and integrated using a Faraday cup and an ELCOR Model A310C Current Indicator and Integrator. The gamma-ray dose rate was measured on a Victoreen Model 555 Roentgen Rate Meter with 100x probe. Doses of both types of radiation were further checked with the Far West Technology Model 401 Radiachromic Reader. The accuracy of calibration of this reader was in turn checked with a series of radiachromic films irradiated at the National Bureau of Standards with known doses of gamma rays. Devices were irradiated in both biased and unbiased conditions. In the biased devices, gate-to-substrate voltages of $+10 \mathrm{~V}$ for the $n$-channels and $-10 \mathrm{~V}$ for the p-channels were applied. Drain-to-source voltages were not applied during irradiation since in actual flight conditions drain currents flow only for a short time during switching and the drain-to-source voltages applied during irradiation are generally known not to have any effects on the gate threshold.

\section{Annealing}

Annealing of the samples was done in a Dewar type oven identical to that used in previous work. ${ }^{6-9}$ The variation in the temperature along the sample array was less than $\pm 1.5^{\circ} \mathrm{C}$. To eliminate short-term room-temperature annealing, the devices were kept at room temperature for 15 minutes after irradiation was stopped and before annealing began. Since all the data were taken at room temperature, annealing had to be interrupted by quickly cooling the samples to room temperature, taking the data, and then rapidly reheating the samples to the annealing temperature. Approximately 12 to 15 minutes elapsed each time between return of the samples to room temperature and the taking of the measurements. This rapid cooling and reheating of the devices produced no observable effects on the threshold potential of the unirradiated samples, as was shown by unirradiated control devices. All the devices were annealed with no gate biases applied during the annealing process since, as shown in a previous work, ${ }^{8}$ in the case of p-channels, gate biases of both polarities alter the rate of annealing: negative biases retard annealing and positive biases lead to instabilities in the threshold potential due to bias-temperature (B-T) treatment effects.

\section{Data Acquisition}

The extent of both radiation damage and annealing was measured by the shift in the threshold potential, $\mathrm{V}_{\mathrm{GT}}$ as defined above. A gate threshold voltage readout and device biasing system built by the University of Michigan for the ground simulation phase of the CREM experiment was used to read out gate threshold voltages before and after irradiation and after each annealing step.

\footnotetext{
*CREM-Component Radiation Effects Measurement experiment is an interagency cooperative program established between NASA, the U.S. Air Force and the Defense Nuclear Agency and is composed of the flight experiment, the ground simulation experiment with monoenergetic radiation sources and the theoretical predictions based on the current models of the space radiation environment. The purpose of the program is to measure radiation effects in semiconductor devices and ICs in the space radiation environment and to compare them with results of the ground simulation experiment and theoretical predictions. Results of one of these experiments, flown on Explorer 55 (Atmosphere Explorer-E) have been published. 12,13
}

\section{Experimental Results: Tempering and Isothermal Annealing}

After irradiation, the devices were subjected to two different annealing regimes: tempering and isothermal annealing. In tempering, the temperature is linearly increased as a function of time and the annealing process is interrupted at equal temperature intervals to take readings. In isothermal annealing, the samples are annealed at a constant temperature, the annealing time being carried out at logarithmically increasing intervals.

Since there were small variations in initial and final (after irradiation) $\mathrm{V}_{\mathrm{GT}}$, the data were normalized to unity in the form of an unannealed fraction remaining after some annealing. It is defined as

$$
\mathrm{N}=\frac{\mathrm{V}_{\mathrm{GT}}(\text { after each annealing step })-\mathrm{V}_{\mathrm{GT}}(\text { before irradiation })}{\mathrm{V}_{\mathrm{GT}}(\text { after irradiation })-\mathrm{V}_{\mathrm{GT}}(\text { before irradiation })}
$$

\section{$\underline{\text { Tempering }}$}

Figure 1 shows the results of tempering on RCA's CD 4007A commercial, or "soft" ICs, where unannealed fraction is plotted against the temperature. Initial readings are at room temperature. The temperature was raised at $200^{\circ} \mathrm{C}$ per hour in 25 -degree steps from $25^{\circ}$ to $450^{\circ} \mathrm{C}$, between which the devices were rapidly cooled to take measurements. The data represent averages of six samples, three irradiated with Co-60 gamma rays and three with $1 \mathrm{MeV}$ Van de Graaff electrons. Both sources were used in order to detect any possible difference in annealing modes of the radiation-induced damage due to these different sources of radiation. No observable difference was detected, hence all the curves were averaged and the root mean square error in $\mathrm{N}$ is approximately $\pm 4 \%$.

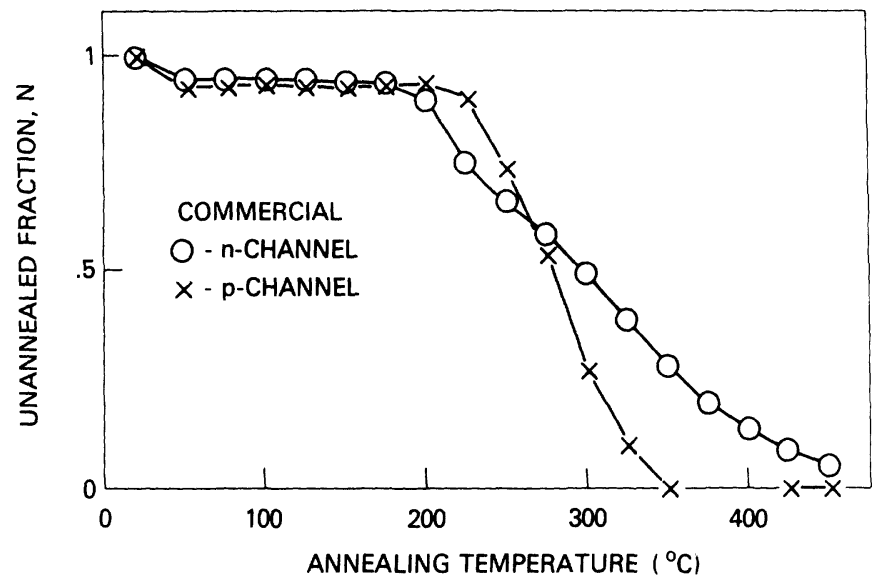

Figure 1. Tempering curves of annealing of radiation-induced damage in $\mathrm{n}$ - and p-channels of RCA's commercial or "soft" CD 4007 CMOS ICs.

As can be seen from the curves, there is a pronounced difference between the $n$ - and $p$-channel tempering curves. There is apparently a more complicated annealing process going on in the n-channels than in the p-channel. This is very strongly reflected in the distributions of the activation energies described below. It is interesting to note that the p-channel tempering curve is very similar in shape to the isochronal curve $^{6}$ obtained earlier on p-channel devices made by the General Micro-electronics Co. several years ago.

A more pronounced difference in the tempering curves between $\mathrm{n}$ - and p-channel devices was found in RCA's J-process devices, as shown in Figures 2 and 3. Figure 2 shows one tempering curve averaged over all the unbiased and biased p-channel transistors (since there was very little difference in the tempering characteristic of these two irradiation modes) and three tempering curves for the unbiased n-channels, irradiated with three different irradiation rates using the Co- 60 source. 
Although the rate effect is small in the case of unbiased n-channels, it is very pronounced in the case of biased n-channels as shown in Figure 3. There, the devices which spent 23.8 hours under irradiation exhibit a comparatively large apparent reverse annealing at the very beginning of the tempering process and then again at about $180^{\circ} \mathrm{C}$, as compared to the devices that were under irradiation for 2.7 and 3.2 hours. No satisfactory explanation for this phenomenon has yet been found. The most probable explanation is that more electrons were trapped in eithe the interface states ${ }^{14}$ or shallow electron traps ${ }^{9}$ in the oxide during the long-term $(23.8 \mathrm{~h})$ exposure to $\mathrm{Co}-60$ radiation under positive gate bias. It is difficult to decide in the light of these experiments alone whether the radiation-induced interface states of shallow oxide electron traps, or maybe both, are responsible for this electron trapping. The lack of change in the transconductances, however, after irradiation for such small radiation doses indicates that there were very few radiationinduced interface states created. Since the total Co- 60 dose in both short- and long-term exposures are the same, the number of electron traps should be the same, which leads one to conclude that the electric field is solely responsible for filling the traps with electrons. (Nothing like that was observed in the unbiased n-channels.) During subsequent heating, the traps release the electrons into the conduction band of silicon, exposing more effective positive charge, which is identified with "reverse" annealing. This large reverse annealing has not been observed in the commercial n-channel devices.

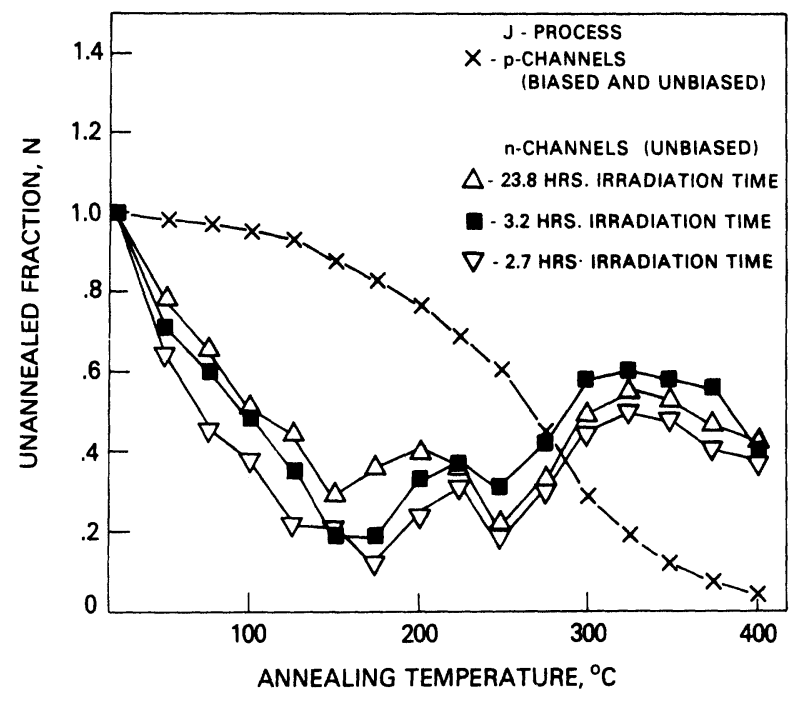

Figure 2. Tempering curves of annealing of radiation-induced damage in n-and p-channels of RCA's J-process CD 4007 CMOS ICs.

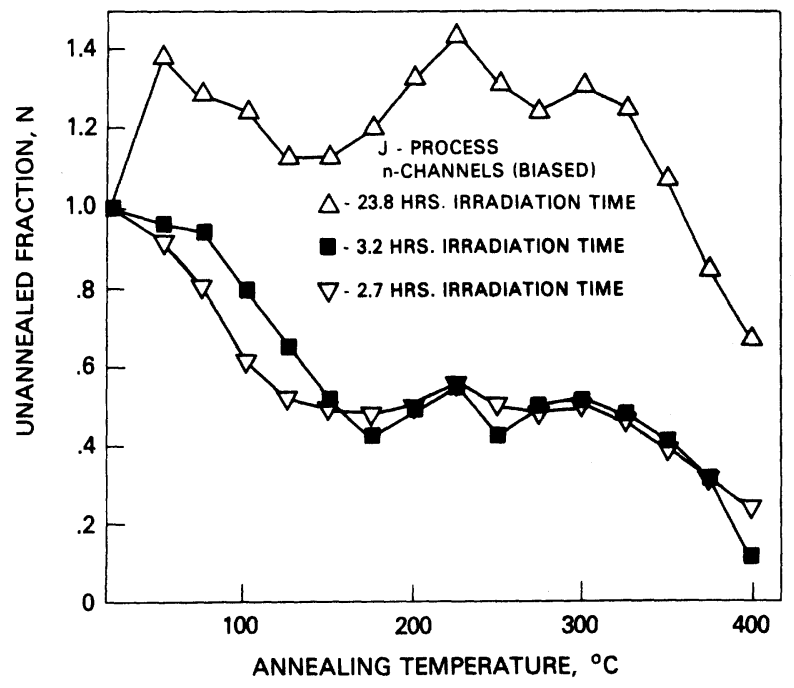

Figure 3. Same as Figure 2, except n-channels are biased during irradiation.
Isothermal Annealing

Isothermal curves were obtained for commercial process devices and their analysis for activation energy of annealing was performed, however, their presentation is beyond the scope of this paper. Of more interest are the isothermal curves of J-process which are represented in Figures 4 and 5 for both p- and n-channels annealed at $25^{\circ}, 125^{\circ}$ and $250^{\circ} \mathrm{C}$. As seen from the curves, the p-channels show an annealing rate which increases monotonically with temperature, as shown by the increasing negative slopes. The n-channels, on the other hand, show considerable annealing at $25^{\circ} \mathrm{C}$ (room temperature), as compared to much less annealing at $125^{\circ} \mathrm{C}$ and $250^{\circ} \mathrm{C}$. This is because there is a considerable reverse annealing at these higher temperatures. In particular, the $250^{\circ} \mathrm{C}$ isothermal shows considerable reverse annealing at the beginning and in the middle of the curve which is to be expected since, in the tempering curves of Figures 2 and 3, this is precisely the region of reverse annealing.

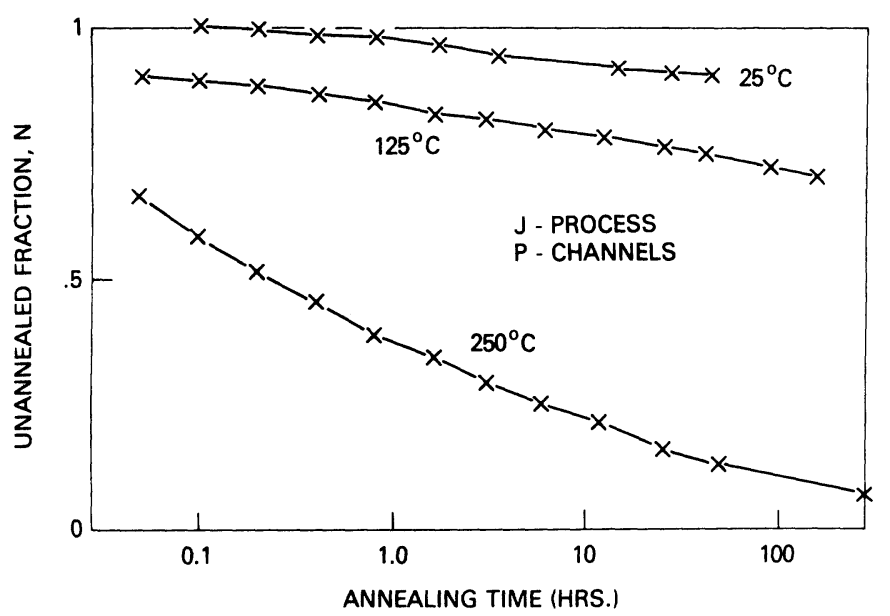

Figure 4. Isothermal curves of annealing of radiation-induced damage in p-channels of RCA's J-process CD 4007 CMOS ICs. The data are an average of both biased and unbiased devices.

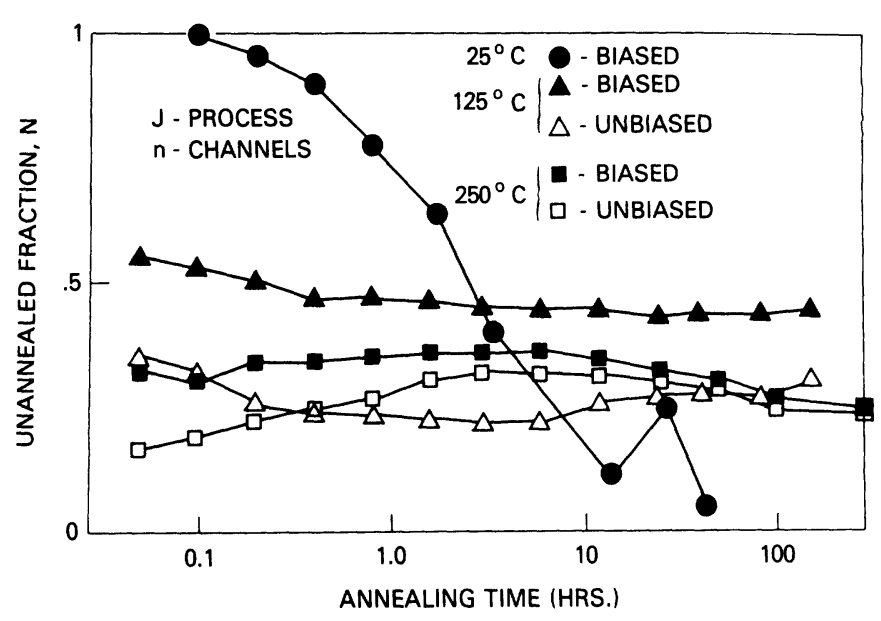

Figure 5. Same as Figure 4, except n-channels.

\section{Activation Energy of the Thermal Annealing Process}

It is generally understood that the increase in the threshold potential of p-channel MOS transistors, or decrease in the case of n-channels, as a result of irradiation is due to the buildup of an excess positive charge in the oxide. We assume in this model that the positive charges are the sinks to which random diffusion of electrons across the potential barriers of silicon-oxide interface occurs during the annealing process. We further assume that an essentially infinite supply of electrons becomes available for recombination with these positive charges. The time 
dependence of the annealing process may then be represented using first-order kinetics as:

$$
\mathrm{dn} / \mathrm{dt}=-\mathrm{Kn}
$$

where $t$ is the annealing time and $\mathrm{n}$ is the density which undergoes the rate process and is, in our case, proportional to the density of effective positive charge. $\mathrm{K}$ is the rate constant which depends on the temperature through the Arrhenius relation:

$$
\mathrm{K}=\mathrm{A} \exp [-\epsilon / \tau(\mathrm{t})],
$$

where $\tau$, which in general may be a function of time, is the product of Boltzmann's constant and the annealing temperature in absolute units, $\mathrm{A}$ is a frequency factor and $\epsilon$ is the activation energy. obtain

Substituting Equation (3) into Equation (2) and integrating, we

$$
n(t)=n_{o} \exp \left\{-A \int_{0}^{t} \exp \left[-\epsilon / \tau\left(t^{\prime}\right)\right] d t^{\prime}\right\} .
$$

When there is a distribution of activation energies, Equation (4) is expressed as:

$$
\mathrm{N}(\mathrm{t})=\int_{0}^{\infty} \mathrm{n}_{\mathrm{o}}(\epsilon) \exp \left\{-\mathrm{A} \int_{0}^{\mathrm{t}} \exp \left[-\epsilon / \tau\left(\mathrm{t}^{\prime}\right)\right] \mathrm{dt}\right\} \mathrm{d} \epsilon,
$$

where $n_{o}(\epsilon)$ is the initial (i.e., before annealing) energy distribution of the defect population density, $\mathrm{n}_{\mathrm{o}}$.

There are two methods to determine $n_{o}(\epsilon)$. The first method is a direct evaluation from experimental results. ${ }^{6}$ The second method is a new approach which we are proposing here and consists of first determining $\mathrm{N}(\mathrm{t})$ from the experimental data, and then determining $\mathrm{n}_{\mathrm{o}}(\epsilon)$ by an integral transform.

In the following, we will derive workable approximations to the solutions of the Equation (5) for both methods and use them to analyze the tempering and isothermal curves for distributions in the activation energies. By doing so, we will compare the two methods.

First Method: Using the appropriate approximation developed by Vand ${ }^{15}$ and refined by Primak, ${ }^{16}$ it can be shown that, for isothermal annealing, the activation energy, $\epsilon_{0}$, is given by:

$$
\epsilon_{\mathrm{o}}=\tau \ln (\mathrm{At})
$$

and the activation-energy distribution is given by:

$$
\mathrm{n}_{\mathrm{o}}\left(\epsilon_{\mathrm{o}}\right) \cong-\mathrm{dN} / \mathrm{dt}(\mathrm{t} / \tau)
$$

similarly, for tempering, the activation energy is given by the transcendental equation:

$$
\epsilon_{\mathrm{o}} / \tau+\ln \left(\epsilon_{\mathrm{o}} / \tau+2\right)=\ln (\operatorname{Ac} \tau)
$$

where $\mathrm{c}$, the tempering constant, is, for our experimental apparatus: $\mathrm{c}=2.087 \times 10^{5} \mathrm{sec} / \mathrm{eV}$, and the activation-energy distribution is given by:

$$
\mathrm{n}_{\mathrm{o}}\left(\epsilon_{\mathrm{o}}\right)=-\mathrm{dN} / \mathrm{d} \tau \cdot 1 /\left(\epsilon_{\mathrm{o}} / \tau+1\right) .
$$

Equations (8) and (9) were used in the analysis of the tempering data of Figures 1-3 to produce the activation energy distributions represented in Figures 6 and 7 and Equations (6) and (7) were used in the analysis of the isothermal data of Figures 4 and 5 . As can be seen from Equations (7) and (9), the activation energy distribution is proportional to the slope of the tempering or isothermal annealing curve; hence, the double maximum in $-\mathrm{dN} / \mathrm{d} \tau$ of the n-channels of the commercial devices gives rise to a double peak in the activation energy distribution, while the single maximum in $-\mathrm{dN} / \mathrm{d} \tau$ of the p-channels of this technology produces a single peak. In the n-channels of the J-process devices the analysis gives rise to even more complicated results. There we observe three maxima in $-\mathrm{dN} / \mathrm{d} \tau$ which gives rise to three peaks in the activation energy distributions. We also observe here some reverse annealing which leads to negative values of the activation energy. This method of analysis is not equipped to handle this latter aspect and thus the regions of negative values of activation energy are not shown. In the analysis of the isothermal curves, each isothermal covers a separate "band" of the activation-energy axis, with some overlap. There are two major difficulties in analyzing the isothermal curves: One is that the slopes are much more difficult to determine, since they are small, and the other is that each isothermal requires a separate group of samples, thus introducing some additional variation in the results. These two factors are responsible for the greater scattering of activation energy values than in the case of tempering analysis. The principal value of the isothermal analysis is for determination of the frequency factor, A, in Eqs. (6) and (8).

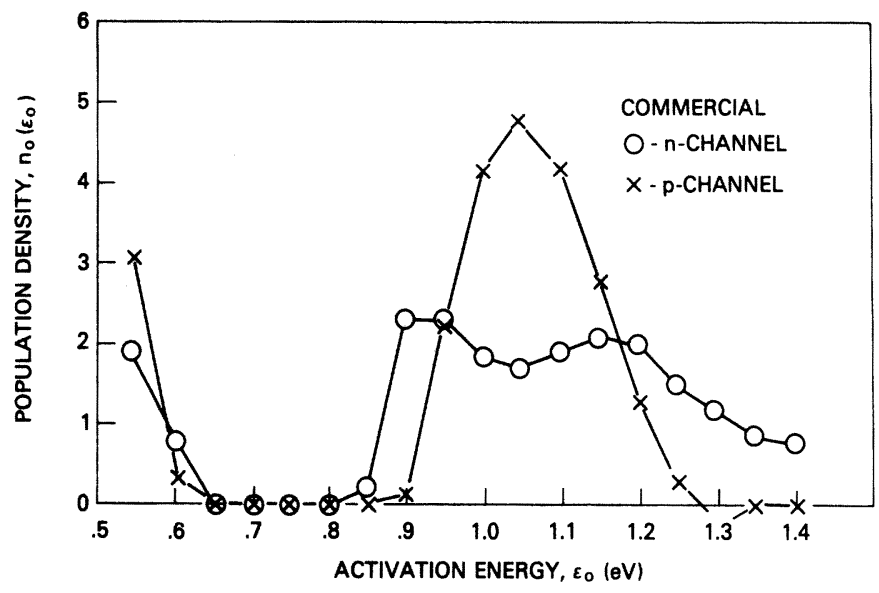

Figure 6. Activation energy distribution calculated from the data shown in Figure 1.

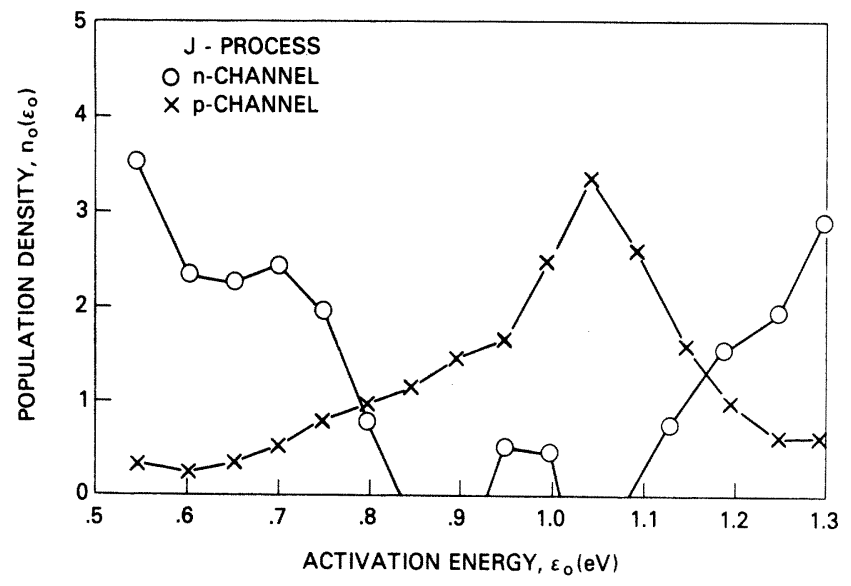

Figure 7. Activation energy distributions calculated from the tempering curves of Figures 2 and 3.

Second Method. In this method we start by assuming that the annealing is described by a reaction kinetics whose order is to be determined from experimental data. By a global fitting which simultaneously amounts to a smoothing procedure, the time $(t)$ dependence of the annealing function is obtained. The distribution function of the activation energy then can be derived directly from the inverse Laplace transform of the annealing function. The mathematical steps will be illustrated by specific CMOS examples. 


\section{(A) p-channel case:}

Because of some essential differences in the annealing characteristics of the p-channel case from that of the n-channel case, we will discuss these cases separately. We will use the data of isothermal annealing at $200^{\circ} \mathrm{C}$ of the $\gamma$-irradiated, unbiased case. Figure 8 shows data for two such devices. The solid curve is obtained from a seventh order reaction kinetics:

$$
n(t)=\left(1+1.38 \times 10^{-4} t\right)^{-1 / 6}
$$

where $t$ is in the units of seconds. The order which is related to the value of the exponent of Eq. (10), is arrived at by curve fitting as an adjustable parameter. According to Equation (3) and (5),

$$
k T \frac{n_{o}(K)}{K}=\int_{c-i \infty}^{c+i \infty} e^{K t} N(t) d t,
$$

where $\mathrm{k}$ is the Boltzman constant and $\mathrm{N}$ is as defined in Equation (1). The solution of this inverse Laplace transform is

$$
\mathrm{n}_{\mathrm{o}}(\mathrm{K})=\frac{\mathrm{C}}{6} \mathrm{x}^{1 / 6} \mathrm{e}^{-\mathrm{x}} / \mathrm{kT} \Gamma(7 / 6),
$$

where $\mathrm{x}=1.38 \times 10^{-4} \mathrm{AK}$. The parameters $\mathrm{A}$, as defined in Eq. (3), and $C$ are adjusted such that the value of the maximum $n_{o}$ and its corresponding $\epsilon_{\mathrm{o}}$ coincide with the spectrum of $\mathrm{n}(\epsilon)$ from the numerical method as given in Figure 2. The results from the two methods are shown in Figure 9. In general these two curves are quite similar. Two discrepancies noted are: (1) The curve from Equation (12) shows a broader distribution at high energies. This can be explained by an approximation that, in the direct Laplace transform upon which Equation (13) is based, the limits of $K$ are assumed to be from zero to infinity, whereas actually they should be from a small but finite value of $\mathrm{K}$ to infinity. (2) There is an anomaly of $\mathrm{n}_{\mathrm{o}}(\epsilon)$ in the low energy region. This effect is not included in the formulation of Equation (11). A proper treatment of this effect will be published elsewhere.

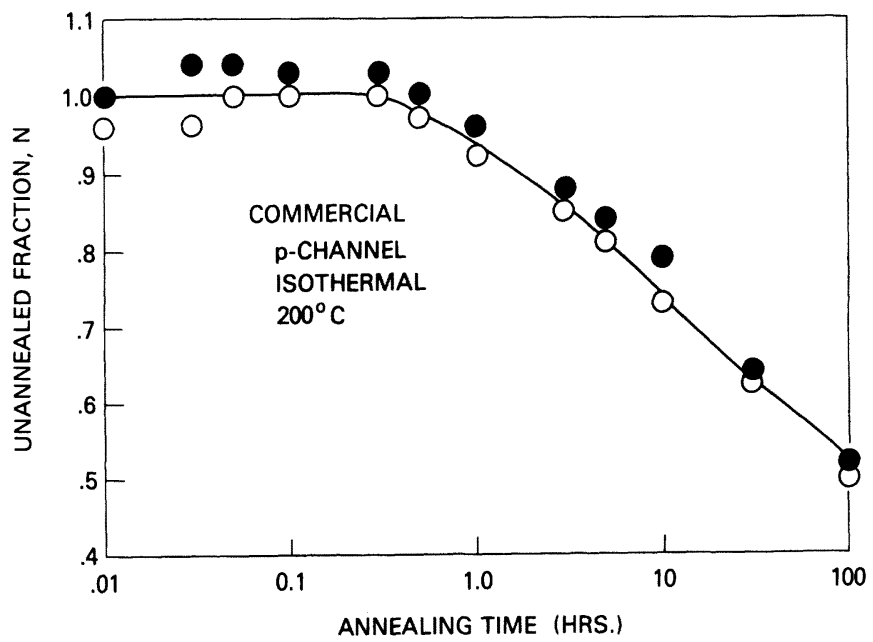

Figure 8. The data points are from individual devices. The solid curve represents Eq. 10.

(B) n-channel case:

The data of isothermal annealing of n-channel devices shows that the recovery consists of two simultaneous processes. Each has its own reaction role, but both processes contribute to the recovery of the threshold gate voltage. This section will discuss such recovery processes. Mathematically, the recovery of the unannealed fraction can be represented by:

$$
N(t)=N_{1} F_{1}(t)+N_{2} F_{2}(t)
$$

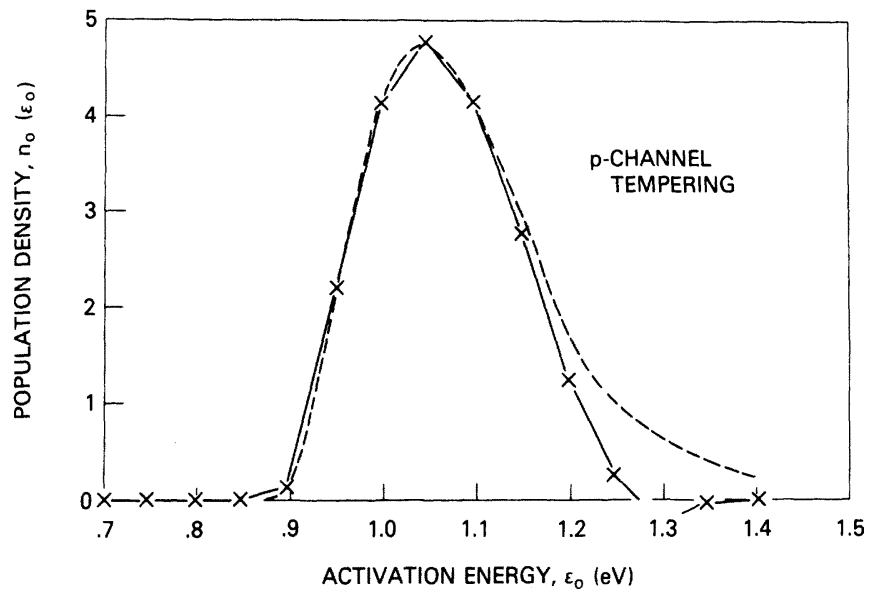

Figure 9. Solid curve is from the numerical method (Figure 6), dotted curve is from the inverse Laplace transform method.

where $F_{1}$ and $F_{2}$ are the recovery functions for the processes and $N_{1}$ and $\mathrm{N}_{2}$ their corresponding concentrations. Such a system has been considered in the literature to describe the recovery in mixed amorphous and crystalline phases or macromolecular mixtures. ${ }^{17}$

One important characteristic of Equation (13) is that it cannot be represented by a usual reaction kinetics such as that of Equation (2) or its extension, nor by an inclusion of multiple terms to the right hand side of Equation (2). The reason is that mathematically, from $\mathrm{N}_{1}$ and $\mathrm{N}_{2}$, one can determine their sum $\mathrm{N}$, but not vice versa, that is, from $\mathrm{N}$, one cannot determine $\mathrm{N}_{1}$ or $\mathrm{N}_{2}$. One manifestation of this limitation is that only in systems such as that of Equation (13), can a multiple activation energy spectrum be obtained.

In the application of Equation (13) to the annealing data, we used a trial and error method to determine $F_{1}$ and $F_{2}$. A satisfactory fitting is obtained when both $F_{1}$ and $F_{2}$ are given by a second order kinetics, that is, $n$ on the right hand side of Equation (2) is to be replaced by $n^{2}$ and $\mathrm{K}$ by $\kappa_{1}$ and $\kappa_{2}$ for the two processes. Thus, Equation (13) becomes

$$
N(t)=\frac{N_{1}}{1+N_{1} \kappa_{1} t}+\frac{N_{2}}{1+N_{2} \kappa_{2} t}
$$

The parameters $\mathrm{N}_{1}, \mathrm{~N}_{2}$ and $\kappa_{1}, \kappa_{2}$ are determined from the data of $200^{\circ} \mathrm{C}\left(473^{\circ} \mathrm{K}\right)$ isothermal annealing of a $\gamma$-radiated $n$-channel under a biased field as shown in Figure 10, where the symbols are for two sets of experimental data and the solid curve is obtained by the following equation:

$$
\mathrm{N}(\mathrm{t})=\frac{0.334}{1+10.1 \mathrm{t}}+\frac{0.666}{1+0.00148 \mathrm{t}}
$$

The fitting is not exact, but the trend of the experimental data is clearly represented.

From Equation (15), we can calculate the spectrum of the activation energy distribution, that is, following the procedure of Equation (5),

$$
\mathrm{n}_{\mathrm{o}}(\epsilon)=\left(0.334 \mathrm{x}_{1} \mathrm{e}^{-\mathrm{x}_{1}}+0.666 \mathrm{x}_{2} \mathrm{e}^{-\mathrm{x}_{2}}\right) / 473 \mathrm{k},
$$

where

$$
\begin{aligned}
& \mathrm{x}_{1}=\frac{\exp (-\epsilon / 473 \mathrm{k}) \mathrm{A}}{10.1}, \\
& \mathrm{x}_{2}=\frac{\exp (-\epsilon / 473 \mathrm{k}) \mathrm{A}}{1.48 \times 10^{-3}}
\end{aligned}
$$




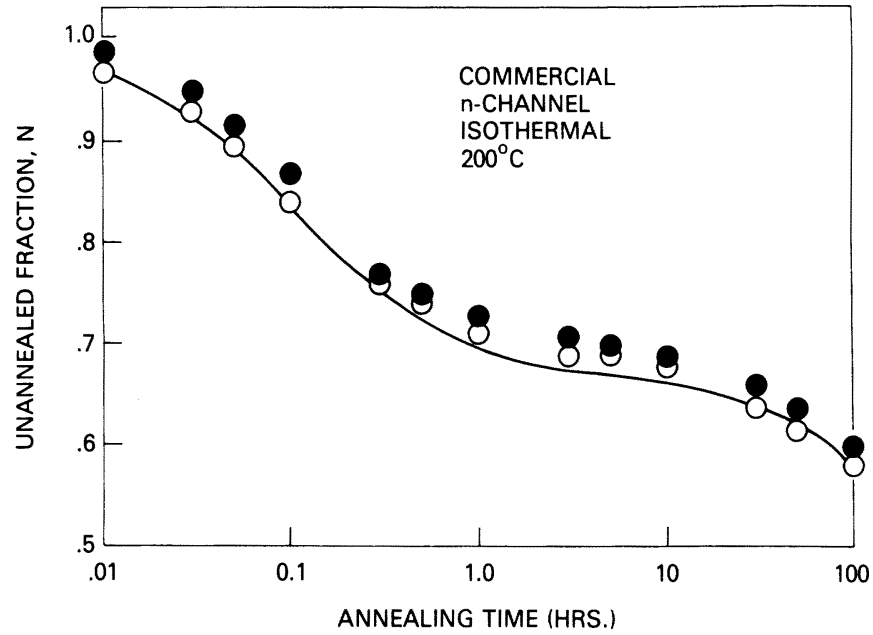

Figure 10. The data points are from individual devices. The solid curve represents Eq. 14.

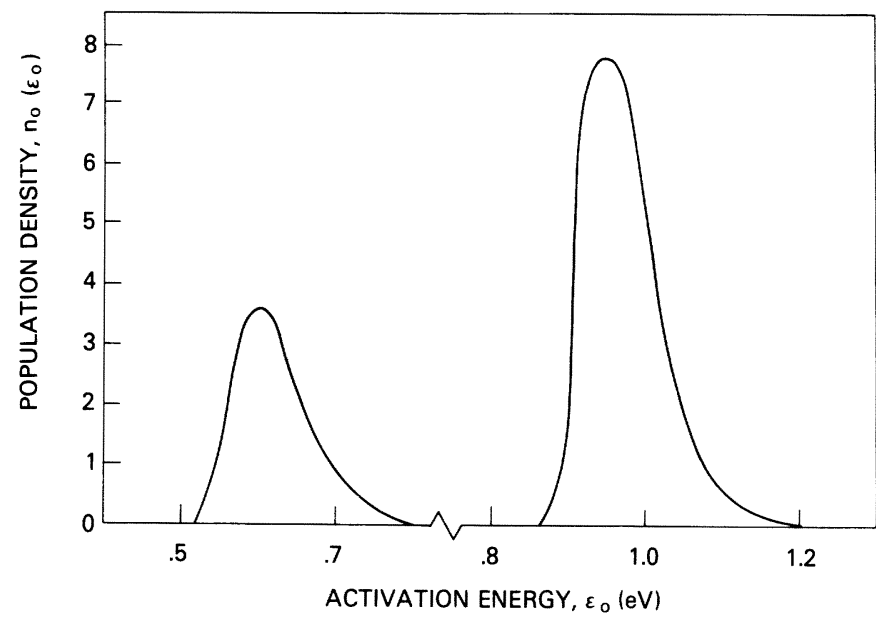

Figure 11. Activation energy distribution calculated from the data represented by solid curve of Figure 10

Following the procedure of Danchenko, et al., ${ }^{6}$ a value of $1 \times 10^{7}$ is adapted for the frequency factor A, and the result is shown in Figure 11. The left peak, which has smaller activation energy, represents a fast recovery, and the higher peak, with larger activation energy, a slow recovery.

\section{Discussion and Conclusions}

The most interesting result of this investigation and analysis is the observation of a pronounced difference in the annealing modes of radiation-induced damage in the $\mathrm{p}$ - and $\mathrm{n}$-channel transistors in semiconductor devices of the same type, fabricated using both commercial and J-process CMOS technologies. It is believed that in the annealing process the radiation-induced positive charges are fixed in the gate oxide and that at elevated temperatures, electrons from the conduction band of silicon diffuse through the interface and oxide potential barriers and recombine with the fixed positive charges. There is some experimental evidence (Figure 2 of Reference 8 ) that this is the mechanism of thermal annealing in the p-channels. Although we have not repeated these experiments on the devices in the present work, we observe that the tempering curves of these commercial devices are very similar in shape to the isochronal curves in the previous work. ${ }^{8}$ The authors are not aware of any experimental data which show that the reverse is true, i.e., that the radiation-induced holes in the oxide are thermally excited and diffuse across the interface to recombine with the electrons in silicon. Further indirect evidence that seems to preclude the latter possibility is the existence of "reverse" annealing in the present experiments. It is difficult to see how heating could induce diffusion of holes from the silicon back into the oxide. The distribution of activation energies may be thought of as the distribution of interface potential barriers over which the thermally excited electrons diffuse into the oxide. It was suggested ${ }^{18}$ that the lowering of the barriers is due to the fact that the tails of the potential wells of the hole traps in the oxide extend across the interface into the silicon. The traps located farther from the interface lower the barrier less than those located closer to the interface (as schematically indicated in Figure 7 of Reference 6); hence the distribution in the activation energies. The electrons are then propelled further into the oxide by the process of hopping 9 from one trap to another. If this is the mechanism for thermal annealing, then the difference between the $\mathrm{p}$ - and $\mathrm{n}$-channels indicate that the radiation-induced charge traps are impurity-dependent and that the traps are preferably formed near the impurities rather than elsewhere in the amorphous oxide. We know that the n-channel gate oxide is grown on the surface of silicon which has been doubly diffused with dopants as compared with that of p-channels. To start with, the n-type silicon wafer, doped with phosphorus, is used in the formation of the p-channel gate oxide. A p-type well is then formed by ion implantation of boron. This is the silicon from which the n-channel gate oxide is grown, containing both phosphorus and boron impurities.

Let us consider the annealing process of Figure 1 and the corresponding distribution of activation energies of Figure 6 for the n-channel device. Initially, at lower temperatures, one type of charge trap is annealed as electrons diffuse into the oxide overcoming comparatively lower barriers. Then, as the temperature is increased, some resistance to annealing is observed until the temperature is high enough for the electrons to overcome a second distribution of barrier heights. These appear to be more difficult to anneal than the traps in the p-channel.

In the n-channels of the J-process the annealing kinetics is even more complicated. There, the data imply not two but three predominant traps, although no third doping impurity was deliberately introduced by the manufacturing process. The analysis of these data is somewhat obscured by the unusually large reverse annealing in the $\mathrm{n}$-channels, but not as much as it would seem at first glance. Since the activation energy analysis depends solely on the slopes of the annealing curves, the activation energy distributions from each of the curves of Figures 2 and 3 would be very similar. However, for a clearer picture, the reverse annealing should be measured by a series of separate experiments and separated from the annealing curves. The initial maximum observed between room temperature and $50^{\circ} \mathrm{C}$ in the tempering curves reflect an additional distribution of low-energy states, most of which probably anneal very rapidly at room temperature immediately after irradiation. Reverse annealing observed in these devices indicates that much of the positive charge trapping in the gate oxide is overcompensated by electron trapping at interface states or by shallow electron trapping in the oxide, hence the devices show an apparent hardness to radiation as a result.

Comparing the two methods of analysis for activation energy distribution, the first method has the advantage of model independence while, in the second method, the solution of $\mathrm{N}(\mathrm{t})$ is dependent on the model. On the other hand, the first method suffers from data scattering, as well as the requirement of a larger number of experiments while the second method already smoothes the data in the determination of $\mathrm{N}(\mathrm{t})$, therefore, $\mathrm{n}_{\mathrm{o}}(\epsilon)$ appears a smooth function and if there is a secondary structure, such as inflections or two peaks, the result from this second method is less ambiguous than that from the first.

It should be pointed out that in this work we have established a distributive nature of activation energies for the specific devices under consideration over a large temperature range $\left(25^{\circ}-450^{\circ}\right)$ and the emphasis was on the shape of the distribution spectrum itself rather than on the absolute values of the activation energies. In our procedure of analysis the absolute values could be shifted to a limited extent by the choice of the frequency factor, A. A detailed discussion of the effect of varying $A$ on the value of the activation energy is beyond the scope 
of this paper and has been published in an earlier work. ${ }^{6}$ The criterion for choosing the value $\mathrm{A}=10^{7}$ in this work is that this value determines the continuity from segment to segment in the envelope of the activation energy distribution, where the various segments are the result of analysis from various isothermals of annealing. Furthermore, the main features of the spectrum (such as peaks) based on the analysis from tempering curves should coincide with those based on the analysis from isothermal curves. Those criteria were satisfied to the best possible fitting with $\mathrm{A}=10^{7}$.

Simons and Hughes ${ }^{2}$ and Derbenwick and Sander ${ }^{5}$ found much smaller activation energies of annealing for MOS devices, i.e., 0.4 and $0.33 \mathrm{eV}$, respectively. Their results support our findings that there is a distribution of activation energies when the annealing is extended to higher annealing temperatures. The temperature range of isochronal annealing employed by Derbenwick ${ }^{5}$ is about $-60^{\circ}-+110^{\circ} \mathrm{C}$ and it was possible to plot experimental points on a straight line as a function of inverse temperature, which led to an indication of a single activation energy of $0.33 \mathrm{eV}$. In our temperature range of annealing $\left(25^{\circ}-450^{\circ} \mathrm{C}\right)$ no such single straight line could be plotted indicating the existence of a distribution of activation energies, and the radiation damage we were investigating just begins to anneal out at temperatures above $100^{\circ} \mathrm{C}$ (Figures 1,2 and 3). An analysis of the $25^{\circ} \mathrm{C}$ isothermal in our devices contributed to the $0.5-0.7 \mathrm{eV}$ part of the spectrum, where most of the annealing processes investigated in Derbenwick ${ }^{5}$ have already taken place.

\section{Acknowledgments}

The authors wish to express their thanks to Dr. Andrew HolmesSiedle for many valuable discussions and to Mr. Lawrence Bromery of the Goddard Space Flight Center Radiation Facility for his valuable assistance and substantial contribution in operating both the $2 \mathrm{MeV}$ Van de Graaff and Co-60 gamma-ray source for these experiments.

\section{References}

1. M. Simons and H. L. Hughes, IEEE Trans. Nucl. Sci., NS-18, 106, (1971).
2. M. Simons and H. L. Hughes, Ibid, NS-19, 282, (1972)

3. M. Simons, Ibid, NS-21, 172, (1974).

4. H. H. Sander and B. L. Gregory, Ibid, NS-22, 2157, (1975).

5. G. F. Derben wick and H. H. Sander, Ibid, NS-24, 2244, (1977).

6. V. Danchenko, U. D. Desai and S. S. Brashears, J. Appl. Phys., 39, No. $5,2417,(1968)$.

7. V. Danchenko and S. S. Brashears, IEEE Trans. Nucl. Sci., NS-15, (1968).

8. V. Danchenko. U. D. Desai, J. M. Killiany and S. S. Brashears, IEEE Trans. Electron Devices, ED-15, 751, (1968).

9. V. Danchenko, J. D. Walker, Jr. and S. S. Brashears, Intern. Conf. on Properties and Use of M.I.S. Structures, Grenoble, France, 413, (1969).

10. B. Maximov and E. M. Reiss, GOMAC, 227, (1978).

11. T. W. Griswold and J. Maserjian, GOMAC, 398, (1978).

12. R. A. Cliff, V. Danchenko, E. G. Stassinopoulos, M. Sing, G. J. Brucker and R. S. Ohanian, IEEE Trans. Nucl. Sci., NS-23, 1781, (1976).

13. E. G. Stassinopoulos, V. Danchenko, R. A. Cliff, M. Sing, G. J. Brucker and R. S. Ohanian, Ibid, NS-24, 2289, (1977).

14. Private communication from Dr. A. Holmes-Siedle.

15. V. Vand, Proc. Phys. Soc. (London) A55, 222 (1943).

16. W. Primak, Phys. Rev., 100, p. 1677 (1955).

17. P. H. Fang, Japan, J. Appl. Phys., 16, p. 1739, (1977).

18. S. R. Hofstein, Solid-State Electr., 10, 657 (1967). 